\title{
ReMed: rapport annuel 2014
}

\section{Comité de direction de ReMed}

\section{ReMed Suisse - Peter Christen, nouveau directeur de programme}

Le réseau de soutien ReMed a un nouveau directeur de programme en la personne du Dr Peter Christen, qui a repris début juillet 2014 les fonctions du Dr Michael Peltenburg, cofondateur de ReMed. Au bénéfice d'une large expérience dans le domaine de la médecine de famille, le Dr Christen est également spécialisé en psychothérapie et en psychosomatique, ce qui fait de lui une personne parfaitement qualifiée pour diriger le programme ReMed. Egalement en juillet, le Dr Franco Gusberti a été élu au Comité de direction. Les responsables de ReMed tiennent à ce que les jeunes médecins, toujours plus nombreux à faire appel au réseau de soutien, soient également représentés dans l'équipe dirigeante. Ils comptent encore élargir en 2015 le Comité de direction à d'autres représentants de la jeune génération de médecins. Actuellement, le Comité de direction se compose des personnes suivantes:

\section{Direction du programme}

- Dr Peter Christen, spécialiste en médecine interne générale, FMH, direction médicale du programme

- Esther Kraft, lic. rer. oec., cheffe de la division Données, démographie et qualité de la FMH, direction administrative du programme

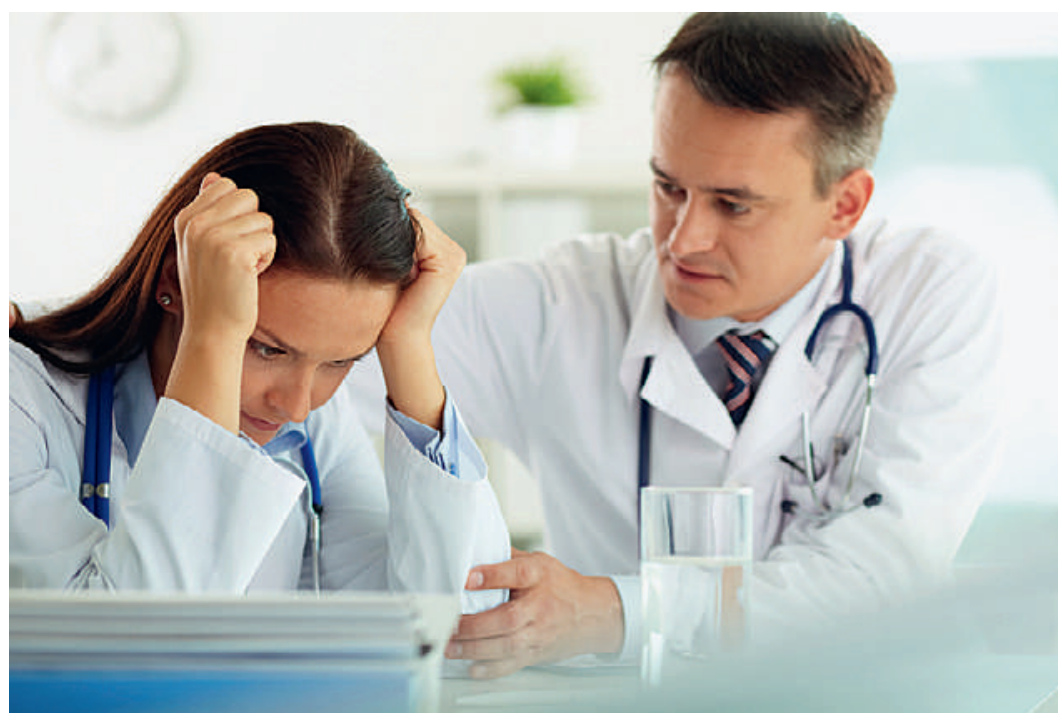

En 2014, ReMed a sensibilisé les hôpitaux à son offre pour la rendre accessible au plus grand nombre de médecins.

\section{Comité de direction}

- Dr Jürg Bänninger, spécialiste en médecine interne générale, FMH

- Dr Peter Birchler, spécialiste en psychiatrie et psychothérapie, FMH

- Dr Franco Gusberti, spécialiste en psychiatrie et psychothérapie, FMH

- Dr Susanne Meier, spécialiste en médecine interne générale, FMH

- Dr Mirjam Tanner, spécialiste en psychiatrie et psychothérapie, FMH

- Dr Michael Peltenburg, spécialiste en médecine interne générale, FMH

\section{Programme ReMed}

Lors de l'année passée sous revue, le Comité de direction a une nouvelle fois profité de sa participation à différents congrès en Suisse (notamment lors du Burn-Out Symposium Kilchberg et du Public Health Congress à Olten) mais aussi à l'étranger (lors de l'International Conference on Physician Health [ICPH] à Londres) pour sensibiliser le corps médical à l'offre de ReMed. A ces occasions, il a recueilli différentes réactions et noué de nombreux contacts avec d'autres organisations, qu'il compte poursuivre. En 2015, ReMed entend participer à de nouvelles manifestations pour présenter son programme et son offre de soutien. En 2014, le réseau a été sollicité plus de 80 fois et, fait intéressant, environ un quart des prises de contact ont eu lieu le jeudi.

\section{Consolider le réseau}

\section{Intervisions Suisse alémanique}

Les rencontres d'intervision qui existent depuis l'introduction du programme en Suisse alémanique ont été reconduites avec succès l'année passée sous revue. Le réseau de confrères qui offrent des prestations de coaching, conseil et thérapie ainsi qu'un soutien professionnel s'est encore consolidé en 2014. ReMed ne donne aucune consigne concernant les thèmes des rencontres d'intervision, l'idée étant de traiter les questions, attentes et demandes des participants concernant des cas particuliers ou des situations plus générales. Le Comité de direction poursuit 
ses efforts en vue d'élargir le réseau et d'y accueillir de nouveaux médecins. Toute personne intéressée est la bienvenue.

\section{Rencontre Romandie}

Les partenaires romands du réseau et les représentants du Comité de direction se sont rencontrés à trois reprises au cours de l'année passée sous revue. Ces rencontres ont permis de mettre sur pied des

\section{Les médecins concernés peuvent contacter ReMed via la ligne d'assistance $24 \mathrm{~h}$ sur 24 ou par courriel.}

pièces de théâtre interactives en Suisse romande, et ont également servi de plateforme d'échange entre les médecins romands actifs dans le domaine du conseil.

\section{Améliorer la sensibilisation}

L'envoi de dépliants et d'affichettes à tous les responsables de la qualité au début 2014 a eu pour but de sensibiliser les hôpitaux à l'offre de ReMed et de la rendre accessible au plus grand nombre de médecins. ReMed a également publié plusieurs témoignages de médecins en situation de crise dans le Bulletin des médecins suisses pour expliquer comment il vient en aide à ces personnes et trouve des solutions. Le témoignage "Agressions sexuelles: ReMed ne juge pas» (BMS 42/2014) a suscité de nombreuses réactions auxquelles ReMed a répondu (BMS 1-2/2015) en présentant de manière nuancée et détaillée le comportement à tenir et les possibilités d'action lors d'abus, en particulier d'ordre sexuel. Le témoignage en question a visé à sensibiliser davantage les médecins à ce thème encore tabou et à faire connaître l'offre préventive de ReMed afin d'inciter les personnes concernées à prendre contact.

\section{Accroître sa visibilité}

A l'instar des années précédentes, plusieurs représentations de la troupe de théâtre interactif «Knotenpunkt» ont eu lieu en 2014. Les représentations à Bâle et au Public Health Congress d'Olten ont connu un important succès et ont suscité un large écho auprès $\mathrm{du}$ public. L'équipe de ReMed se félicite également d'avoir pu mettre sur pied une offre équivalente pour la Suisse romande avec la troupe "Le Caméléon». La première représentation qui s'est tenue à Neuchâtel en décembre 2014 a été très appréciée du public. Grâce à l'intervention ciblée d'un animateur, ces pièces de théâtre interactives permettent aux spectateurs d'influer selon leur propre ressenti sur la manière d'agir des différents personnages. Le public intervient activement sur ce qui se passe sur scène et teste en direct sa vision des choses. Il voit ainsi immédiatement les conséquences de ses propres actions.

\section{Poursuivre le développement}

Le programme national ReMed, le Comité de direction et les partenaires du réseau continueront à apporter leur soutien aux médecins en situation de crise en 2015. Les médecins concernés peuvent contacter ReMed via la ligne d'assistance 24 h sur 24 (080o o 73633) ou par courriel à help[at]swissremed.ch. Une équipe de conseillers expérimentés prendra contact avec eux dans les 72 heures pour s'entretenir de leur situation.

En 2015, ReMed continuera également à proposer des rencontres d'intervision, à organiser des représentations de théâtre interactif en Suisse romande et en Suisse alémanique, à participer à des manifestations et congrès et à publier des témoignages afin de faire connaître son offre au plus grand nombre. 